\title{
OPEN Immune checkpoint inhibitor combination therapies very frequently induce secondary adrenal insufficiency
}

Katsunori Manaka ${ }^{1}$, Junichiro Sato ${ }^{1}$, Maki Takeuchi ${ }^{1}$, Kousuke Watanabe ${ }^{2}$, Hidenori Kage ${ }^{2}$, Taketo Kawai ${ }^{3}$, Yusuke Sato ${ }^{3}$, Takuya Miyagawa ${ }^{4}$, Daisuke Yamada ${ }^{4}$, Haruki Kume ${ }^{3}$, Shinichi Sato ${ }^{4}$, Takahide Nagase ${ }^{2}$, Taroh liri ${ }^{1,5}$, Masaomi Nangaku ${ }^{1} \&$ Noriko Makita $^{1 凶}$

Immune checkpoint inhibitors (ICls) are potent therapeutic options for many types of advanced cancer. The expansion of ICls use however has led to an increase in immune-related adverse events (irAEs). Secondary adrenal insufficiency (AI) can be life-threatening especially in patients with delayed diagnosis. We retrospectively investigated secondary $\mathrm{Al}$ in $\mathrm{ICl}$-treated patients. A total of 373 cancer patients treated with ICls were included and evaluated. An adrenocorticotropic hormone (ACTH) deficiency was described in 13 patients. Among 24 patients with a combination of nivolumab and ipilimumab therapy, 7 patients (29\%) developed secondary Al in a median time of 8 weeks during the combination therapy and 2 of 15 patients (13\%) developed isolated ACTH deficiency during maintenance nivolumab monotherapy following the combination therapy. More than half of the patients (4/7) with a combination therapy-induced multiple anterior hormone deficiencies was diagnosed as secondary AI based on regular ACTH and cortisol tests with slight subjective symptoms. Secondary Al can arise frequently and rapidly in cancer patients receiving a combination ICI therapy, and thus we speculate active surveillance of Al using regular ACTH and cortisol tests during the combination therapy might be useful for avoiding life-threatening conditions due to secondary Al.

ICI-induced endocrine dysfunction can involve the thyroid, parathyroid, pituitary, adrenal gland, and the pancreas $^{1-5}$. Although Immune checkpoint inhibitor (ICI) therapies are often discontinued in patients with severe immune-related adverse events (irAEs) in non-endocrine organs such as the lung, colon, liver and kidney, patients with irAEs involving endocrine organs can continue their ICI regimens if adequate hormone replacement therapy is given ${ }^{4-10}$. Some endocrine disorders such as ACTH deficiency caused by pituitary irAEs (i.e. secondary adrenal insufficiency $[\mathrm{AI}]$ ) and fulminant type 1 diabetes mellitus (DM), are life-threatening conditions, especially when the diagnoses are delayed. For the early diagnosis of type 1 DM during ICI use, patient education, routine glucose monitoring, and self-measurements of blood glucose levels are recommended ${ }^{11}$. In the case of secondary AI however, in which the ACTH levels cannot appropriately increase against the low levels of cortisol, most presenting symptoms such as fatigue, appetite loss, and joint pain are non-specific, often resulting in a delayed diagnosis ${ }^{1-3,12}$.

In our current study, we addressed some of the pertinent clinical questions regarding the adequate diagnosis of secondary AI induced by ICI therapies in daily clinical practice. We suspected whether routine ACTH and cortisol tests might be helpful for the early diagnosis of secondary AI, whether certain symptoms or laboratory data could indicate secondary AI prior to conducting ACTH and cortisol tests, and what might be done to diagnose secondary AI in daily practice. In our current study, we retrospectively surveyed ICI-induced secondary $\mathrm{AI}$ in a cohort from our hospital.

\footnotetext{
${ }^{1}$ Department of Nephrology and Endocrinology, Graduate School of Medicine, The University of Tokyo, 7-3-1 Hongo, Bunkyo-ku, Tokyo 113-8655, Japan. ${ }^{2}$ Department of Respiratory Medicine, Graduate School of Medicine, The University of Tokyo, Tokyo, Japan. ${ }^{3}$ Department of Urology, Graduate School of Medicine, The University of Tokyo, Tokyo, Japan. ${ }^{4}$ Department of Dermatology, Graduate School of Medicine, The University of Tokyo, Tokyo, Japan. ${ }^{5}$ Department of Pharmacology, St. Marianna University School of Medicine, Kawasaki, Japan. ${ }^{\square}$ email: norimaki-tky@umin.ac.jp
} 


\section{Results}

Baseline profiles of the study subjects. A total of 373 patients (269 men and 104 women) who received ICI therapies at our hospital were included in this study (Table 1). The median age of this cohort was 68 years (range 23-86 years). The cancers targeted by the ICI regimens in this population included head and neck cancer, esophageal cancer, gastric cancer, lung cancer, kidney cancer, bladder cancer and upper urinary tract (renal pelvis and ureter) urothelial carcinoma, malignant melanoma, and other malignancies (malignant pleural mesothelioma, pancreatic cancer, Hodgkin's lymphoma, and cancer of unknown primary). The patients who received a combination therapy of ipilimumab and nivolumab had stage 4 kidney cancer (renal cell carcinoma) or stage 4 malignant melanoma.

Twenty-nine of our current study patients had pre-existing hypothyroidism due to chronic thyroiditis, the use of tyrosine-kinase inhibitors, or a thyroidectomy. One patient had hypopituitarism due to a history of craniopharyngioma. Four patients had an adrenal insufficiency due to glucocorticoid use, bilateral adrenal metastases, and a bilateral adrenalectomy. Seventy-two patients were receiving drugs for DM.

Endocrine immune-related adverse events other than pituitary ones. Although beyond the main focus of this study, we noted that thyroid irAE developed in 37 patients, adrenalitis (primary AI) developed in one patient, and fulminant type 1 diabetes mellitus developed in 3 patients in our current cohort (Table 2).

Serious adverse events other than endocrine ones. ICIs were discontinued due to serious irAEs in 60 of our current study patients (a cumulative total of 61 patients). Interstitial pneumonia was the most common irAE resulting in the cessation of ICI treatments (31 patients). We were concerned that the frequency of serious irAEs might increase in the patients with AI due to an acceleration of autoimmune responses. We noted however that the patients with ICI-induced AI showed no statistically significant increase in such severe complications, compared to patients without ICI-induced AI (Fisher's exact test; $p=0.25$; odds ratio 2.16 ( $95 \%$ confidence interval 0.48-7.82)).

Adverse events in the pituitary gland. Secondary AI arose in 13 of the study patients (Tables 1 and 3). Eight of these patients showed multiple anterior pituitary hormone deficiencies, which include ACTH deficiency, and remaining 5 patients showed an isolated ACTH deficiency. Multiple anterior pituitary hormone deficiency occurred in 7 patients who underwent a combination therapy of ipilimumab and nivolumab, and in 1 patient who was administered ipilimumab after nivolumab (Table 1). Isolated ACTH deficiency occurred in 2 patients receiving pembrolizumab alone, 2 patients treated with nivolumab after a combination therapy of ipilimumab and nivolumab, and 1 patient who was administered pembrolizumab after nivolumab alone and ipilimumab alone (Table 1). Hence, 9 of 24 patients (38\%) who received a combination therapy of ipilimumab and nivolumab developed secondary AI. It developed during the combination therapy in 7 of these patients $(7 / 24$; $29 \%$ ) and during early courses of maintenance nivolumab therapy after four courses of combination therapy in the 2 remaining patients (Tables 1 and 3, Fig. 1). In contrast, none of our $212(0 \%)$ study patients receiving nivolumab alone without history of the combination therapy, only 3 of our 119 (3\%) study patients receiving pembrolizumab alone, and 1 of $8(13 \%)$ patients administered ipilimumab alone developed secondary AI (Table 1). In addition to the high frequency of secondary AI in patients who received the combination therapy, most secondary AI developed during the combination therapy. MRI analysis revealed enlargement of the pituitary gland in 6 of the $8(75 \%)$ patients with multiple anterior hormone deficiency (i.e. hypophysitis) and revealed no enlargement in pituitary gland in any patient with isolated ACTH deficiency (Table 3, Fig. 2). One patient without swelling in the pituitary gland underwent an MRI at 3 weeks after the diagnosis of anterior pituitary hormone deficiency (patient No 4) and the other 7 patients received an MRI within 2 weeks of this diagnosis. Headache symptoms were very characteristic of the 5 patients with pituitary swelling (Fig. 2, patients No. 1, 3, 5-7; 83\%).

In our present study cohort, the plasma ACTH levels and serum cortisol levels were measured at every outpatient visit in the patients undergoing combination therapy for malignant melanoma (regular checkup) and when there was a suspicion of AI from symptoms and signs. The casual serum cortisol levels, which were checked regularly or to work up the possibility of secondary AI, were low $(2.8 \mu \mathrm{g} / \mathrm{dL}$ at highest) in the morning or under stressful conditions with inappropriately low or normal levels of ACTH (Fig. 3a). To evaluate the pituitaryadrenal axis and diagnose possible secondary AI, loading tests were sequentially performed. Except patient No. 4,10 , and 12, 10 of the 13 patients in our present series diagnosed with an ACTH deficiency using a loading test with corticotropin releasing hormone (CRH), an endogenous ACTH secretagogue, were examined for adrenal function by single ACTH loading. This indicated a reduced cortisol response in all 10 patients (Fig. 3b, c). At first, we suspected that concomitant adrenalitis might have developed in secondary AI patients because a rapid ACTH loading test was performed soon after the ACTH deficiency developed. However, subsequent prolonged ACTH loading tests revealed a preserved cortisol response in 5 of the secondary AI patients (Table 3: patients No. 1-3, 6, and 7) who showed a reduced cortisol response to the single ACTH loading.

Investigation of factors related to secondary adrenal insufficiency. It would be very clinically useful to be able to diagnose secondary AI based on abnormalities in routine laboratory data. It is well known in this regard that hyponatremia and/or eosinophilia are signs of AI and it has been reported that thyroid stimulating hormone (TSH) deficiency often develops in patients with ipilimumab-induced hypophysitis ${ }^{13}$. As thyrotoxic patients due to thyroid irAEs also show low TSH levels, we investigated TSH levels with free thyroxine $\left(\mathrm{fT}_{4}\right)$ levels. Fisher's exact tests revealed that new-onset eosinophilia and low TSH levels with normal or low $\mathrm{fT}_{4}$ levels were related to secondary AI (odds ratio $6.6(p<0.01)$ and $22.1(p<0.01)$, respectively) (Table 4$)$. There was no 


\begin{tabular}{|c|c|c|c|c|c|c|}
\hline ICI regimens used & $\begin{array}{l}\text { Number of treated } \\
\text { patients }^{\mathrm{a}}\end{array}$ & Age & Sex & Primary site & Prior ICI therapies & $\begin{array}{l}\text { Secondary adrenal } \\
\text { insufficiency }\end{array}$ \\
\hline \multirow[b]{3}{*}{ Nivo } & \multirow[b]{3}{*}{224} & \multirow{3}{*}{$\begin{array}{l}\text { Median } 66 \\
{[23-86]} \\
\text { Mean } 64.3\end{array}$} & \multirow[b]{3}{*}{$\begin{array}{l}\text { M } 166 \\
\text { F } 58\end{array}$} & \multirow{3}{*}{\begin{tabular}{|l|} 
Stomach 60 \\
Esophagus 29 \\
Kidney 35 \\
Melanoma 25 \\
Lung 23 \\
Head and neck 51 \\
Others 4
\end{tabular}} & None 211 & $0 / 211$ \\
\hline & & & & & Nivo + Ipi 15 & $2 / 15$ \\
\hline & & & & & Pembro 1 & $0 / 1$ \\
\hline \multirow[b]{2}{*}{ Pembro } & \multirow[b]{2}{*}{119} & \multirow[b]{2}{*}{$\begin{array}{l}\text { Median } 70 \\
{[26-86]} \\
\text { Mean 67.9 }\end{array}$} & \multirow[b]{2}{*}{$\begin{array}{l}\text { M } 82 \\
\text { F } 37\end{array}$} & \multirow{2}{*}{\begin{tabular}{|l} 
Bladder and upper \\
urinary tract 48 \\
Lung 44 \\
Melanoma 12 \\
Head and neck 10 \\
Kidney 2 \\
Others 4
\end{tabular}} & \begin{tabular}{|l|} 
None 115 \\
\end{tabular} & $2 / 115$ \\
\hline & & & & & Ipi 4 & $1 / 4$ \\
\hline \multirow{2}{*}{ Ipi } & \multirow{2}{*}{$8^{\mathrm{b}}$} & \multirow{2}{*}{$\begin{array}{l}\text { Median } 66.5 \\
{[42-86]} \\
\text { Mean } 63.4\end{array}$} & \multirow{2}{*}{$\begin{array}{l}\text { M } 5 \\
\text { F } 3\end{array}$} & \multirow{2}{*}{ Melanoma 8} & Nivo 7 & $1 / 7$ \\
\hline & & & & & Pembro 1 & $0 / 1$ \\
\hline \multirow{3}{*}{ Anti-PD-L1 agents } & \multirow{3}{*}{29} & \multirow{3}{*}{$\begin{array}{l}\text { Median } 74 \\
{[48-81]} \\
\text { Mean } 70.7\end{array}$} & \multirow{3}{*}{$\begin{array}{l}\text { M } 21 \\
\text { F } 8\end{array}$} & \multirow{3}{*}{$\begin{array}{l}\text { Lung } 27 \\
\text { Merkel Cell carci- } \\
\text { noma } 2\end{array}$} & None 24 & $0 / 24$ \\
\hline & & & & & Nivo 2 & $0 / 2$ \\
\hline & & & & & Pembro 3 & $0 / 3$ \\
\hline \multirow{2}{*}{ Nivo + Ipi } & \multirow{2}{*}{24} & \multirow{2}{*}{$\begin{array}{l}\text { Median } 67 \\
{[40-81]} \\
\text { Mean } 64.7\end{array}$} & \multirow{2}{*}{$\begin{array}{l}\text { M } 16 \\
\text { F } 8\end{array}$} & \multirow{2}{*}{\begin{tabular}{|l} 
Kidney 16 \\
Melanoma 8
\end{tabular}} & None 22 & $6 / 22$ \\
\hline & & & & & Nivo 2 & $1 / 2$ \\
\hline
\end{tabular}

Table 1. Number of treated patients and ICI regimens used. ICI, immune checkpoint inhibitor; nivo, nivolumab; pembro, pembrolizumab; ipi, ipilimumab, M, male; F, female. (a) Some patients were treated with multiple regimens and the "Number of treated patients" is therefore greater than the actual number of patients $(n=373)$. (b) All 8 cases treated with ipilimumab alone had undergone a first-line therapy with anti-PD-1 antibodies (nivolumab or pembrolizumab).

\begin{tabular}{|c|c|c|c|c|c|}
\hline \multirow[b]{2}{*}{ Category } & \multirow[b]{2}{*}{$\begin{array}{l}\text { Number of } \\
\text { patients }\end{array}$} & \multicolumn{4}{|l|}{ Ongoing use } \\
\hline & & $\begin{array}{l}\text { Anti-PD-1 } \\
\text { antibody } \\
\text { Nivo (total } \\
209) \\
\text { Pembro (total } \\
\text { 116) }\end{array}$ & $\begin{array}{l}\text { Anti-CTLA-4 } \\
\text { antibody (total 8) }\end{array}$ & $\begin{array}{l}\text { Anti-PD-1 + CTLA-4 } \\
\text { antibodies (total 24) }\end{array}$ & $\begin{array}{l}\text { Anti-PD-L1 antibody } \\
\text { (total 30) }\end{array}$ \\
\hline Thyroid & 37 & & & & \\
\hline Hypothyroidism & 23 & 18 & 2 & & 3 \\
\hline Subclinical hypothyroidism & 7 & 5 & 1 & & 1 \\
\hline Transient thyrotoxicosis & 7 & 5 & & 2 & \\
\hline Pituitary & 13 & & & & \\
\hline Isolated ACTH deficiency & 5 & $\begin{array}{l}5 \text { (nivo 2, } \\
\text { pembro 3) }\end{array}$ & & & \\
\hline $\begin{array}{l}\text { Multiple anterior pituitary } \\
\text { hormone deficiency }\end{array}$ & 8 & & 1 & 7 & \\
\hline Adrenal & 1 & & & & \\
\hline Primary adrenal insufficiency & 1 & 1 & & & \\
\hline \multicolumn{6}{|l|}{$D M$} \\
\hline Fulminant type $1 \mathrm{DM}$ & 3 & 2 & & 1 & \\
\hline
\end{tabular}

Table 2. Endocrine irAEs and ICI regimens used. ACTH, adrenocorticotropic hormone; ICI, immune checkpoint inhibitor; nivo, nivolumab; pembro, pembrolizumab; DM, diabetes mellitus.

significant association between hyponatremia and secondary AI (Table 4). None of these variables was found to be both highly sensitive and specific for this condition.

\section{Discussion}

We have here retrospectively analyzed cancer patients treated with ICIs at our hospital and found that a combination therapy with ipilimumab and nivolumab can cause secondary AI at a surprisingly high frequency. The incidence of secondary AI was as high as $29 \%(7 / 24)$ in the patients receiving a combination therapy and as $13 \%$ $(2 / 15)$ in the patients under a maintenance therapy just after ICI combination therapy (Table 3). Meanwhile, no patients who received nivolumab therapy without history of the combination therapy $(0 / 212)$ developed secondary AI. It seems that the frequency of secondary AI during nivolumab maintenance therapy just after the 


\begin{tabular}{|c|c|c|c|c|c|c|c|c|c|c|c|c|c|}
\hline \multirow[b]{2}{*}{ Patient no } & \multirow[b]{2}{*}{ Age } & \multirow[b]{2}{*}{ Sex } & \multirow[b]{2}{*}{ Cancer } & \multirow[b]{2}{*}{$\begin{array}{l}\text { At onset of } \\
\text { SAI }\end{array}$} & \multirow[b]{2}{*}{$\begin{array}{l}\text { Weeks at } \\
\text { diagnosis }\end{array}$} & \multicolumn{2}{|c|}{\begin{tabular}{|l|} 
Pattern of \\
secondary AI
\end{tabular}} & \multirow[b]{2}{*}{$\begin{array}{l}\text { Pituitary } \\
\text { swelling w/c } \\
\text { headache }\end{array}$} & \multicolumn{2}{|c|}{ Symptoms and signs (SS) } & \multicolumn{2}{|l|}{ Casual levels } & \multirow[b]{2}{*}{$\begin{array}{l}\text { Other } \\
\text { irAEs }\end{array}$} \\
\hline & & & & & & IAD & \begin{tabular}{|l} 
Multiple \\
hormone \\
deficiencies
\end{tabular} & & $\begin{array}{l}\text { Except } \\
\text { headache }\end{array}$ & \begin{tabular}{|l|}
$\begin{array}{l}\text { Serum } \\
\text { sodium } \\
(\mathrm{mmol} / \mathrm{L})\end{array}$ \\
\end{tabular} & $\begin{array}{l}\text { ACTH (pg/ } \\
\mathrm{mL})\end{array}$ & $\begin{array}{l}\text { Cortisol } \\
(\mu \mathrm{g} / \mathrm{dL})\end{array}$ & \\
\hline 1 & 50 & M & RCC & Nivo + ipi & 9 & & $\begin{array}{l}\bullet \\
\text { ACTH, } \\
\text { TSH, PRL }\end{array}$ & $\stackrel{\bullet}{\text { Headache }}$ & $\begin{array}{l}\text { Fatigue, } \\
\text { appetite } \\
\text { loss, nausea, } \\
\text { eosinophilia }\end{array}$ & 139 & 3.2 & 0.3 & $\begin{array}{l}\text { Transient } \\
\text { thyrotoxi- } \\
\text { cosis }\end{array}$ \\
\hline 2 & 75 & M & RCC & Nivo + ipi & 8 & & $\begin{array}{l}\mathbf{Q}_{\mathrm{ACTH}}, \mathrm{Gn}, \\
\mathrm{PRL}\end{array}$ & - & \begin{tabular}{|l|} 
Fatigue, \\
appetite loss, \\
eosinophilia
\end{tabular} & 141 & 2.7 & 0.2 & \\
\hline 3 & 40 & $F$ & MM & Nivo + ipi & 6 & & АCTH, TSH & $\begin{array}{l}\bullet \\
\text { Headach } \\
\text { (continuous) }\end{array}$ & Eosinophilia & 142 & 15.1 & 1.0 & $\begin{array}{l}\text { Fluminant } \\
\text { Type } 1 \mathrm{DM}\end{array}$ \\
\hline 4 & 63 & M & MM & Nivo + ipi & 5 & & $\begin{array}{l}\bullet \\
\text { ACTH, TSH, } \\
\text { Gn }\end{array}$ & - Headache & $\begin{array}{l}\text { Fatigue, } \\
\text { eosinophilia }\end{array}$ & 141 & 1.3 & 1.4 & \\
\hline 5 & 77 & $\mathrm{~F}$ & MM & Nivo + ipi & 15 & & АСТH, TSH & $\begin{array}{l}\bullet \\
\text { Headache } \\
\text { (continuous) }\end{array}$ & $\begin{array}{l}\text { Appetite } \\
\text { loss, pain in } \\
\text { joints }\end{array}$ & 139 & 2.0 & 0.6 & \\
\hline 6 & 81 & F & MM & Nivo + ipi & 7 & & \begin{tabular}{|l|} 
\\
ACTH, TSH, \\
Gn, PRL, \\
GH
\end{tabular} & $\stackrel{\bullet}{\text { Headache }}$ & $\begin{array}{l}\text { appetite loss, } \\
\text { fatigue }\end{array}$ & 131 & 4.6 & 0.5 & \\
\hline 7 & 66 & M & MM & Nivo + ipi & 12 & & $\begin{array}{l}\text { ACTH, } \\
\text { TSH, Gn, } \\
\text { PRL, GH }\end{array}$ & Headache & $\begin{array}{l}\text { Fatigue, } \\
\text { nausea, } \\
\text { hypotension }\end{array}$ & 133 & 2.6 & 0.3 & Enteritis \\
\hline 8 & 55 & M & RCC & $\begin{array}{l}\text { Nivo (main- } \\
\text { tenance Tx) }\end{array}$ & 12 & $\bullet$ & & - & \begin{tabular}{|l|} 
Hiccup, \\
appetite loss, \\
weakness, \\
hypona- \\
tremia
\end{tabular} & 110 & 1.6 & 0.6 & Diarrhea \\
\hline 9 & 76 & M & RCC & $\begin{array}{l}\text { Nivo (main- } \\
\text { tenance Tx) }\end{array}$ & 18 & $\bullet$ & & - & \begin{tabular}{|l|} 
Fatigue, \\
appetite loss, \\
eosinophilia
\end{tabular} & 136 & 3.5 & 0.3 & \\
\hline 10 & 73 & M & Bladder & Pembro & 27 & $\bullet$ & & Not tested & \begin{tabular}{|l|} 
Appetite \\
loss, vomit- \\
ing,
\end{tabular} & 134 & 1.2 & 0.3 & \\
\hline 11 & 74 & M & Bladder & Pembro & 18 & 0 & & Not tested & $\begin{array}{l}\text { Septic shock, } \\
\text { hyper- } \\
\text { kalemia, } \\
\text { hypoglyce- } \\
\text { mia }\end{array}$ & 134 & 8.1 & 2.2 & \\
\hline 12 & 42 & M & MM & Pembro & 65 & $\bullet$ & & - & \begin{tabular}{|l|} 
Fatigue, \\
nausea, \\
eosinophilia
\end{tabular} & 137 & 9.0 & 2.8 & $\begin{array}{l}\text { Thyroiditis } \\
\text { on ultra- } \\
\text { sound }\end{array}$ \\
\hline 13 & 86 & M & MM & Ipi & 32 & & $\bullet_{\mathrm{ACTH}, \mathrm{Gn}}$ & $\bullet$ & \begin{tabular}{|l|} 
Fatigue, \\
appetite loss, \\
hypogly- \\
cemia, \\
eosinophilia, \\
diarrhea
\end{tabular} & 142 & 1.0 & 1.3 & \\
\hline
\end{tabular}

Table 3. Detailed clinical profiles of the patients with ICI-induced secondary AI. Four patients (No 3-6) were diagnosed their SAI based on the results of regular ACTH and cortisol tests. A TSH deficiency was diagnosed based on TSH, fT4, and/or a thyrotropin-releasing hormone (TRH) loading test. ACTH, adrenocorticotropic hormone; TSH, thyroid stimulating hormone; Gn, gonadotropin; PRL, prolactin; GH, growth hormone; M, male; F, female; RCC, renal cell carcinoma; MM, malignant melanoma; Bladder, bladder carcinoma; combination, combination therapy of ipilimumab and nivolumab; nivo, nivolumab; pembro, pembrolizumab; ipi, ipilimumab; cs, courses; IAD, Isolated ACTH deficiency; SAI, secondary adrenal insufficiency; SS, symptoms and signs; DM, diabetes mellitus.

combination therapy was high compared to nivolumab monotherapy. As for time to develop secondary AI, we cannot discuss statistical difference of it between the combination therapy group (median: 8 weeks) and monotherapy group (median: 22.5 weeks) because there may be period bias between routine ACTH and cortisol test patients and non-routine test patients in the combination therapy group (Fig. 1). Based on previous reports showing that the median time to develop pituitary irAEs from initial injection of ICIs is 9 weeks with ipilimumab ${ }^{14}$, 4.9 months with nivolumab and 3.3 months with pembrolizumab ${ }^{7}$, we speculate that a combination therapy might cause pituitary irAEs at least as early as a monotherapy with ipilimumab. 


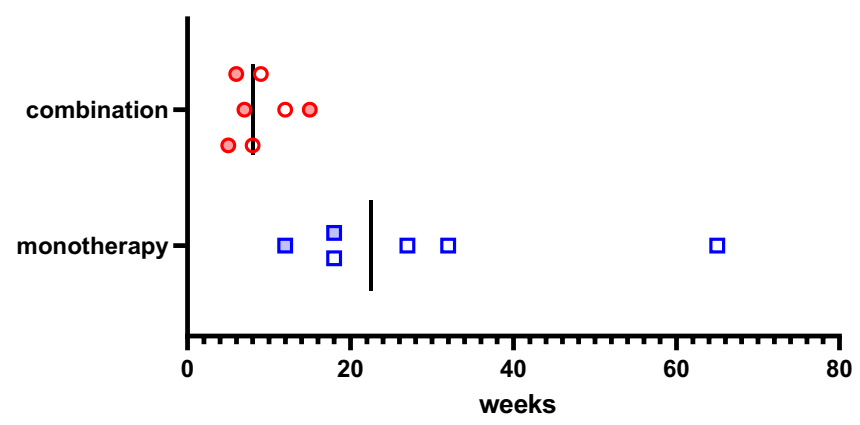

Figure 1. Time to develop secondary adrenal insufficiency (SAI) from initial injection of ICIs in 13 patients. In the combination therapy group, 7 patients developed secondary AI in a median time of 8 weeks during nivolumab and ipilimumab combination therapy. In the monotherapy group, 6 patients developed secondary $\mathrm{AI}$ in a median time of 22.5 weeks during nivolumab, pembrolizumab or ipilimumab monotherapy. In the combination therapy group, filled red circles show the timings of SAI diagnosis based on regular ACTH and cortisol tests, and open red circles show the timings of SAI diagnosis based on symptoms. In the monotherapy group, filled blue squares show the timings of SAI diagnosis during maintenance nivolumab monotherapy following the combination therapy, and open blue squares show the timings of SAI diagnosis during pembrolizumab or ipilimumab monotherapy without history of a combination therapy.

When we focused on the regimens under which ICI-induced secondary AI developed, all of the multiple anterior pituitary hormone deficiencies in our current cohort (8 patients) had developed in patients receiving an anti-CTLA-4 antibody treatment (including ICI combination therapy patients) ${ }^{15}$, and all isolated ACTH deficiencies (5 patients) had developed in patients treated with anti-PD-1 antibody alone (Table 3 ). This particular pattern of pituitary irAEs (hypophysitis with multiple hormone deficiencies vs. isolated ACTH deficiency) was consistent with the findings of previous studies ${ }^{16-19}$. This pattern is also supported by MRI images (hypophysitis with swelling of pituitary gland vs. isolated ACTH deficiency without swelling; Fig. 2, Table 3).

We speculated on the reasons why secondary AI was frequently diagnosed in our study patients receiving a combination ICI therapy. First, in malignant melanoma patients receiving this combination therapy, routine measurements of ACTH and cortisol levels, in addition to as TSH and $\mathrm{fT}_{4}$ levels, had been performed. Notably, 4 patients (patients No 3-6) among the 7 patients who were diagnosed with secondary AI under a combination ICI therapy were diagnosed based on a routine ACTH and cortisol test (Table 3). In the other 3 subjects (patients No 1, 2 and 7), the ACTH and cortisol levels might not have been checked, depending on the extent of the signs and symptoms. Kobayashi et al. also reported that the frequency of ipilimumab-induced hypophysitis was as high as $24 \%$ in their prior prospective study ${ }^{20}$. Hence, active surveillance may have contributed to the increased incidence of secondary AI with an ICI combination therapy in our current cohort. Given the likelihood that secondary AI during an ICI combination therapy develops as frequently as thyroid irAEs for which routine monitoring is recommended in guidelines ${ }^{4,6,9}$, active surveillance of AI using regular ACTH and cortisol tests, at least during the combination therapy, may be a desirable and reasonable approach considering the costs and benefits although no clinical guidelines have recommended it so far ${ }^{4,5,7-10}$.

Second, in addition to the increased incidence of secondary AI detected by active surveillance, we suspect that secondary AI may develop more frequently in Japanese patients than previously thought. We speculate that the genetic background of the Japanese population might affect the frequent incidence in pituitary irAE. A pilot study has described human leukocyte antigen (HLA)-DQB1 ${ }^{\star} 06: 01$, HLA-DPB1 ${ }^{\star} 09: 01$, and HLA-DRB5 ${ }^{\star} 01: 02$ as a risk allele for anti-PD-1 antibody induced pituitary irAE, and HLA-C ${ }^{\star} 03: 03$ as a protective allele for this condition $^{21}$. Another study has suggested that HLA DR15, B52 and Cw12 may be predictive markers for pituitary irAE ${ }^{22}$. Considering that CTLA-4 expressed in the pituitary gland may be a trigger of ipilimumab-induced hypophysitis via classical complementary activation ${ }^{15}$, a polymorphism in the CTLA-4 gene might be responsible for pituitary irAE, similar to autoimmune thyroid disease $\mathrm{e}^{23}$.

We paid attention to headache in our patients as they may be an important early indicator of secondary AI caused by hypophysitis with pituitary swelling (Table 3). It has been reported previously that headache and fatigue are the most common presenting symptoms in ipilimumab containing therapy-induced secondary $\mathrm{AI}^{2,19,24,25}$. We surmise from our present observations that headache may be more specific to secondary AI whereas fatigue is a non-specific symptom often seen in cancer patients. It has been reported that more than a third of patients with pituitary adenoma complain of headache and that $60 \%$ of patients with lymphocytic hypophysitis commonly present with headache before pituitary hormone changes can be characterized ${ }^{26}$. Focusing on the 24 patients who received a combination ICI therapy (Table 1), 6 of the 7 patients with multiple anterior hormone deficiency complained of headache whereas no patient with isolated ACTH deficiency (0/2), nor any of the patients without pituitary hormone dysfunction (0/15), reported this symptom during ICI therapies. In contrast, 5 of 7 patients with multiple anterior hormone deficiency, both patients with isolated ACTH deficiency, and 8 of 15 patients without pituitary hormone dysfunction complained of fatigue during ICI therapies. In addition, continuous headache for several days was noted in 2 patients with hypophysitis before pituitary dysfunction was confirmed using hormonal tests (patients No. 3 and 5). Headache, especially when continuous, may thus be an early specific sign of ICI-induced hypophysitis. Besides headache, we investigated other factors that could be related to 


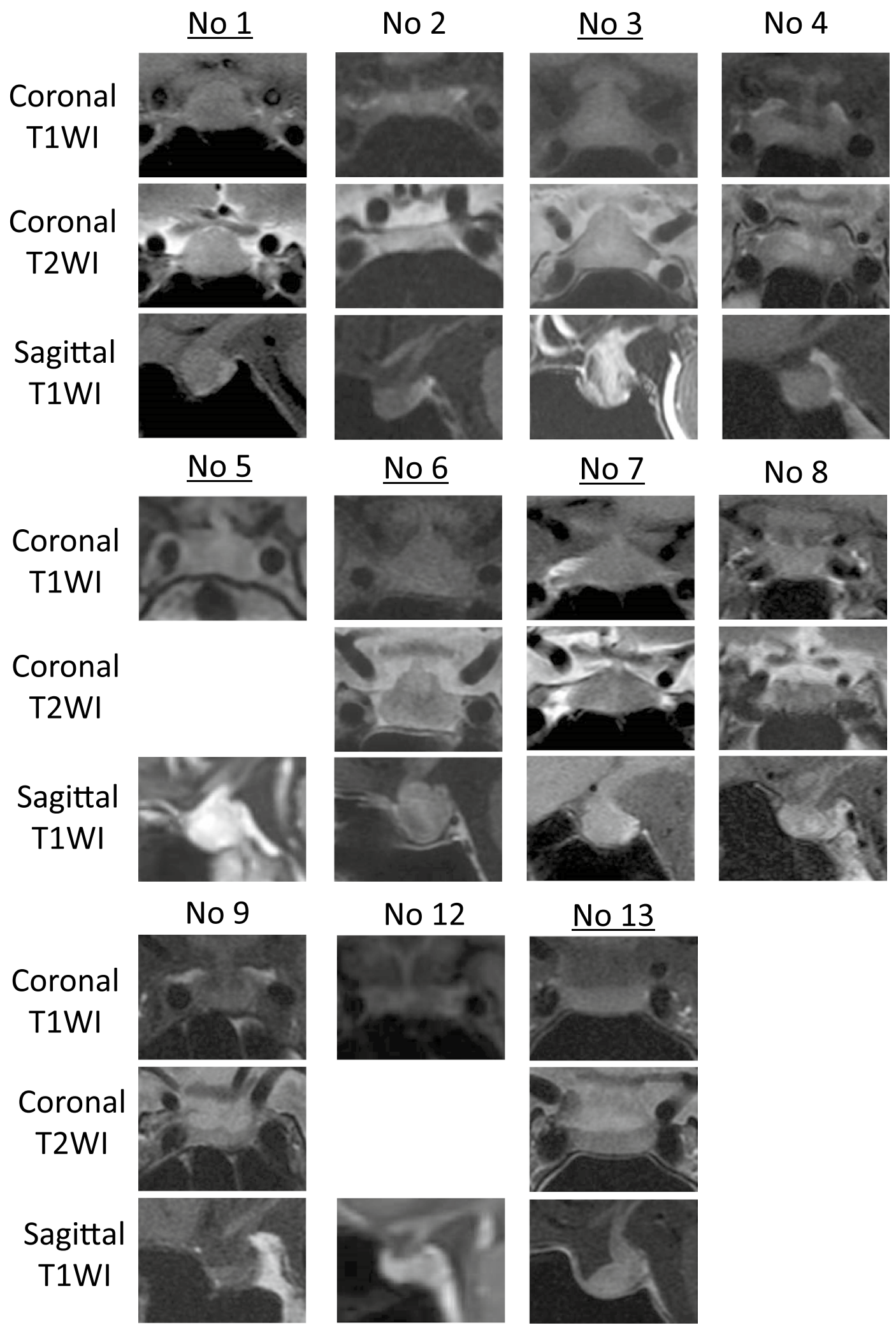

Figure 2. Pituitary MRI of the patients with secondary adrenal insufficiency. MRI analysis revealed enlargement of the pituitary gland in patients No 1,3,5-7, and 13 (highlight with underline). Top panels are coronal non-enhanced T1WI, middle panels are coronal T2WI (patents No 5 and 12 lack coronal T2WI), and bottom panels are sagittal enhanced T1 (if not available, non-enhanced T1WI) for each patient. 

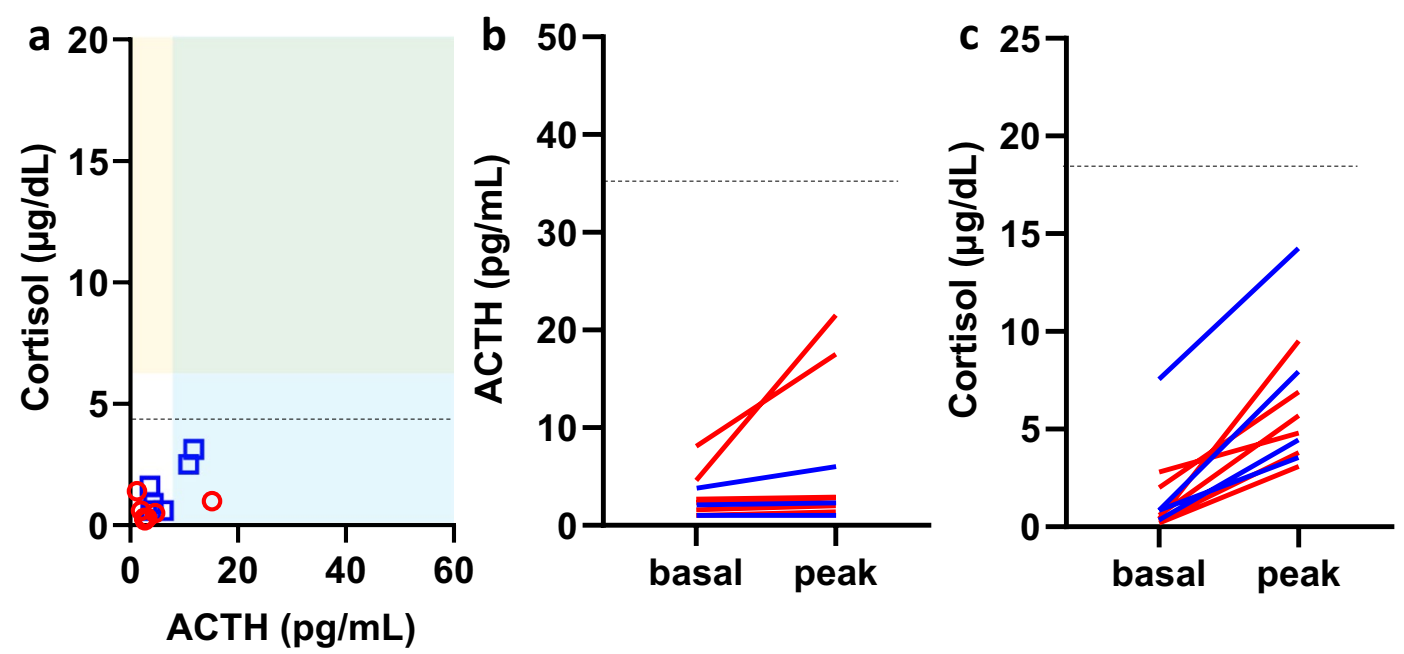

Figure 3. ACTH and cortisol levels upon the suspicion of secondary adrenal insufficiency (AI) and loading test results for the hypothalamus-pituitary-adrenal axis. (a) Casual plasma ACTH levels and serum cortisol levels on regular tests or upon the suspicion of a secondary AI are presented in the scatter plot. Open red circles reflect data of patients in the combination therapy group, and open blue squares reflect data of patients in the monotherapy group. The blue zone shows the reference interval for the plasma ACTH level in the morning $(7.2-63.3 \mathrm{pg} / \mathrm{mL})$, and the yellow zone shows the interval for the serum cortisol level in the morning (7.07$19.6 \mu \mathrm{g} / \mathrm{dL}$ ). Serum cortisol levels were all decreased to below $4.0 \mu \mathrm{g} / \mathrm{dL}$ (dotted line) without elevated plasma ACTH levels. (b) A corticotropin releasing hormone (CRH) test was conducted in which synthetic human $\mathrm{CRH}(100 \mu \mathrm{g})$ was intravenously injected and blood samples were collected before injection and at 30, 60, 90, and $120 \mathrm{~min}$ afterwards. The baseline and peak ACTH levels are shown. Red lines reflect results of patients in the combination therapy group, and blue lines reflect results of patient in the monotherapy group. The cut-off peak value of ACTH $(35 \mathrm{pg} / \mathrm{mL})$ is indicated by the dotted line. (c) A rapid ACTH test was performed in which synthetic ACTH (1-24) at $250 \mu \mathrm{g}$ was intravenously injected. Blood samples were collected prior to the injection and at 30 and 60 min afterwards. The baseline and peak cortisol levels are shown. Red lines reflect results of patients in the combination therapy group, and blue lines reflect results of patient in the monotherapy group. The cut-off peak value of cortisol $(18 \mu \mathrm{g} / \mathrm{dL})$ is indicated by the dotted line.

\begin{tabular}{|l|r|r|l|l|l|}
\hline \multirow{2}{*}{ New-onset } & \multicolumn{2}{|l|}{ AI } & \multicolumn{1}{l|}{} & \\
\cline { 2 - 4 } & + & - & Sensitivity & Specificity & OR (95\% CI) ${ }^{\text {a }}$ \\
\hline Eosinophilia & 8 & 63 & 0.62 & 0.81 & $6.6(1.83-26.5), P<0.01$ \\
\hline+ & 5 & 261 & & & \\
\hline- & 5 & 58 & 0.39 & 0.81 & $2.7(0.68-9.9), p=0.14$ \\
\hline Hyponatremia & 8 & 254 & & & \\
\hline+ & 5 & 8 & 0.38 & 0.97 & $22.1(4.64-100.4), P<0.01$ \\
\hline- & 8 & 292 & & & \\
\hline Low TSH levels with normal or low fT4 levels & \\
\hline+ & 5 &
\end{tabular}

Table 4. Detailed clinical profiles of the patients with ICI-induced secondary AI. OR: odds ratio, CI: confidence interval. ${ }^{a}$ Fisher's exact tests were performed to compare the incidence of secondary AI.

secondary AI and that may also be useful early markers of this condition. New-onset eosinophilia and low TSH levels with normal or low $\mathrm{fT}_{4}$ levels showed an association with secondary AI, but were neither highly sensitive nor specific factors (Table 4). Hyponatremia, which is also frequently shown in AI, showed no significant association with secondary AI. Hyponatremia had low positive productive value (0.079) and could be caused by many other common conditions, such as infection, appetite loss, and dehydration.

It is known that the function of the cortisol secreting cells in the adrenal gland is maintained by ACTH secreted from the pituitary. It has been reported that when ACTH is deficient for one week after pituitary surgery, cortisol secreting cells start to be functionally suppressed ${ }^{27-29}$. In such patients, the adrenal gland cannot react to single ACTH loading, but is recovered by continuous ACTH loading ${ }^{12}$. In our current cohort, we originally suspected that primary AI onset coincided with that of secondary AI because rapid ACTH loading test performed 
soon after developing symptoms of AI had indicated a reduced cortisol response (Fig. 3c). However, a subsequent prolonged ACTH loading test indicating a normal cortisol response ruled out the coincidence of primary AI. Based on our present series, low serum cortisol levels (cortisol $<4.0 \mu \mathrm{g} / \mathrm{dL}$ ) in the early morning or under a stressful condition with inappropriately low or normal levels of ACTH might have enough diagnostic value for ICI-induced secondary AI. Given that a rapid onset ACTH deficiency in ICI-induced secondary AI may be a risk factor for adrenal crisis, reflected by the occurrence of grade 4 irAEs in two of our present patients (patient No. 11, septic shock; patient No 8, severe hyponatremia; Tables 2 and 3), we again contend that an earlier diagnosis of $\mathrm{AI}$ is a key to avoiding life-threatening conditions due to it.

This study had several limitations of note. Principal among these were its retrospective and single center design, small number of included patients, and the heterogeneity of the target cancers and ICIs used. In our cohort, although the number of patients who receive combination therapy of ipilimumab and nivolumab was small, the incidence of secondary AI was much higher than in previous reports. Considering the combination therapy might be approved for additional indications against various cancers, management for a secondary AI will become more important. Our current findings may provide useful information in the meantime that can assist with strategies to reduce severe endocrine-related irAEs.

\section{Materials and methods}

Subjects. All 373 patients who were treated with immune checkpoint inhibitors (ICIs) at our hospital between 1 April 2014 and 31 July 2020 were retrospectively evaluated. The ICI regimens used in this population included nivolumab $(\mathrm{n}=224)$, pembrolizumab $(\mathrm{n}=119)$, ipilimumab $(\mathrm{n}=8)$, atezolizumab $(\mathrm{n}=18)$, durvalumab $(n=10)$, avelumab $(n=1)$, and a combination of nivolumab and ipilimumab $(n=24)$ (Table 1$)$.

This study was approved by The University of Tokyo Hospital Research Ethics Committee (Comprehensive approval of retrospective studies: authentication number 2879-(7)). Informed consent was obtained in the form of opt-out on the web-site and no written informed consent was required to access the medical records, since this study was a retrospective study without any intervention. This study adheres to the ethical principles outlined in the Declaration of Helsinki as amended in 2013 and the Ethical Guidelines for Medical and Health Research Involving Human Subjects in 2017.

Data collection. We retrospectively collected the following data for our study patients: age, sex, history of drug use (including ICIs, glucocorticoids, levothyroxine, and glucose lowering agents), cancer type, imaging results (pituitary MRI), concomitant disease, and blood test results (serum sodium, potassium, chloride, calcium, albumin, phosphorus, creatinine, casual plasma glucose, HbAlc, immune reactive insulin (IRI), c-peptide (CPR), antiglutamic acid decarboxylase (anti-GAD) antibody, ACTH, cortisol, $\mathrm{TSH}, \mathrm{fT}_{4}$, free triiodothyronine $\left(\mathrm{fT}_{3}\right)$, growth hormone $(\mathrm{GH})$, insulin-like growth factor-1 (IGF-1), luteinizing hormone $(\mathrm{LH})$, follicle stimulating hormone (FSH), estradiol, free testosterone, total testosterone, prolactin, plasma renin activity, serum aldosterone concentration, and dehydroepiandrosterone-sulfate (DHEA-S)). All results of blood tests were collected from baseline (before ICI injection) to last administration course of ICI.

Definitions of hyponatremia, eosinophilia, low TSH, and low $\mathrm{fT}_{4}$. Hyponatremia was defined as a serum sodium concentration lower than $135 \mathrm{mmol} / \mathrm{L}$ and eosinophilia as a count of not less than 500 eosinophils per microliter of blood. TSH was measured with enzyme immunoassay using ST AIA-PACK TSH (TOSOH, Ltd., Tokyo, Japan) on AIA-2000 (TOSOH, Ltd., Tokyo, Japan). FT $_{4}$ was measured with fluorimetric enzymelinked immunoassay using ST AIA-PACK FT4 (TOSOH, Ltd., Tokyo, Japan) on AIA-2000 (TOSOH, Ltd., Tokyo, Japan). Low TSH was defined as TSH value below the lower limit of the reference range (TSH reference range $0.38-4.31 \mu \mathrm{IU} / \mathrm{mL}$ ) and low $\mathrm{fT}_{4}$ was defined as $\mathrm{fT}_{4}$ value below the lower limit of the reference range ( $\mathrm{fT}_{4}$ reference range $0.82-1.63 \mathrm{ng} / \mathrm{dL}$ ).

Methods and assessments of loading tests. Corticotropin-releasing hormone (CRH) loading test. One ampule of corticorelin (human, $100 \mu \mathrm{g}$, Nipro ES Pharma Co., Ltd., Osaka, Japan) was intravenously injected and ACTH and cortisol levels were measured at baseline, and at 30,60, 90, and $120 \mathrm{~min}$. The plasma ACTH level at peak after loading corticorelin was increased to 2-3-fold higher than baseline and also above $35 \mathrm{pg} / \mathrm{mL}$ in patients with normal pituitary function (normal response). A cortisol level above $18 \mu \mathrm{g} / \mathrm{dL}$ at peak after loading coticorelin revealed that the patient had a normal adrenal function in addition to a normal pituitary function.

Rapid ACTH loading test. One ampule of tetracosactide acetate (250 $\mu \mathrm{g}$, Daiichi Sankyo Company, Ltd., Tokyo, Japan.) was administered intravenously and the cortisol levels were measured at baseline, and at 30 and $60 \mathrm{~min}$. The serum cortisol level after loading tetracosactide acetate was generally higher than $18 \mu \mathrm{g} / \mathrm{dL}$ (normal response). A reduced cortisol response $(<18 \mu \mathrm{g} / \mathrm{dL})$ to tetracosactide acetate indicated the possibility of either primary or secondary AI.

Prolonged ACTH loading test. One ample of tetracosactide acetate zinc suspension (500 $\mu$ g, Daiichi Sankyo Company, Ltd., Tokyo, Japan.) was administered intramuscularly once per day over 3 consecutive days and 24-h urine free cortisol levels and serum cortisol levels were measured at baseline and after administration. The cutoff value for a diagnosis of a reduced adrenal response was a lower than 2-fold increase in 24-h urine free cortisol excretion, compared to baseline, after three administrations of tetracosactide. 
Differential diagnosis of adrenal insufficiency using a prolonged ACTH loading test. A prolonged ACTH loading test enables a differential diagnosis of AI through the response of cortisol. A no/low response of cortisol in this test indicates primary adrenal deficiency induced by adrenal damage. A positive response of cortisol however indicates secondary AI induced by an ACTH deficiency.

Statistical analysis. The relationships between AI (13 patients of secondary AI and one patient of primary AI, i.e. adrenalitis) and other serious irAEs were examined using a chi-squared test. All statistical analysis was performed using R 3.5.2 software (The R Foundation of Statistical Computing; www.r-project.org). A $p$ value $<0.05$ was considered to indicate statistical significance.

\section{Data availability}

The datasets generated during and/or analyzed during the current study are available from the corresponding author on reasonable request.

Received: 11 February 2021; Accepted: 20 May 2021

Published online: 02 June 2021

\section{References}

1. Gonzalez-Rodriguez, E. \& Rodriguez-Abreu, D. Immune checkpoint inhibitors: Review and management of endocrine adverse events. Oncologist 21, 804-816. https://doi.org/10.1634/theoncologist.2015-0509 (2016).

2. Cukier, P., Santini, F. C., Scaranti, M. \& Hoff, A. O. Endocrine side effects of cancer immunotherapy. Endocr. Relat. Cancer 24, T331-T347. https://doi.org/10.1530/ERC-17-0358 (2017).

3. Byun, D. J., Wolchok, J. D., Rosenberg, L. M. \& Girotra, M. Cancer immunotherapy-immune checkpoint blockade and associated endocrinopathies. Nat. Rev. Endocrinol. 13, 195-207. https://doi.org/10.1038/nrendo.2016.205 (2017).

4. Brahmer, J. R. et al. Management of immune-related adverse events in patients treated with immune checkpoint inhibitor therapy: American Society of Clinical Oncology Clinical Practice Guideline. J. Clin. Oncol. 36, 1714-1768. https://doi.org/10.1200/JCO. 2017.77.6385 (2018).

5. Arima, H. et al. Management of immune-related adverse events in endocrine organs induced by immune checkpoint inhibitors: Clinical guidelines of the Japan Endocrine Society. Endocr. J. 66, 581-586. https://doi.org/10.1507/endocrj.EJ19-0163 (2019).

6. Champiat, S. et al. Management of immune checkpoint blockade dysimmune toxicities: A collaborative position paper. Ann. Oncol. 27, 559-574. https://doi.org/10.1093/annonc/mdv623 (2016).

7. Sznol, M. et al. Endocrine-related adverse events associated with immune checkpoint blockade and expert insights on their management. Cancer Treat Rev 58, 70-76. https://doi.org/10.1016/j.ctrv.2017.06.002 (2017).

8. Puzanov, I. et al. Managing toxicities associated with immune checkpoint inhibitors: Consensus recommendations from the Society for Immunotherapy of Cancer (SITC) Toxicity Management Working Group. J. Immunother. Cancer 5, 95. https://doi.org/10.1186/ s40425-017-0300-z (2017).

9. Girotra, M. et al. The current understanding of the endocrine effects from immune checkpoint inhibitors and recommendations for management. JNCI Cancer Spectrum 2, pky021. https://doi.org/10.1093/jncics/pky021 (2018).

10. Higham, C. E. et al. Society for Endocrinology Endocrine Emergency Guidance: Acute management of the endocrine complications of checkpoint inhibitor therapy. Endocr. Connect. 7, G1-G7. https://doi.org/10.1530/EC-18-0068 (2018).

11. Clotman, K., Janssens, K., Specenier, P., Weets, I. \& De Block, C. E. M. Programmed cell death-1 inhibitor-induced type 1 diabetes mellitus. J. Clin. Endocrinol. Metab. 103, 3144-3154. https://doi.org/10.1210/jc.2018-00728 (2018).

12. Charmandari, E., Nicolaides, N. C. \& Chrousos, G. P. Adrenal insufficiency. Lancet 383, 2152-2167. https://doi.org/10.1016/ S0140-6736(13)61684-0 (2014).

13. De Sousa, S. M. C. et al. Fall in thyroid stimulating hormone (TSH) may be an early marker of ipilimumab-induced hypophysitis. Pituitary 21, 274-282. https://doi.org/10.1007/s11102-018-0866-6 (2018).

14. Min, L. et al. Systemic high-dose corticosteroid treatment does not improve the outcome of ipilimumab-related hypophysitis: A retrospective cohort study. Clin. Cancer Res. 21, 749-755. https://doi.org/10.1158/1078-0432.CCR-14-2353 (2015).

15. Iwama, S. et al. Pituitary expression of CTLA-4 mediates hypophysitis secondary to administration of CTLA-4 blocking antibody. Sci. Transl. Med. 6, 230ra245. https://doi.org/10.1126/scitranslmed.3008002 (2014).

16. Faje, A. Immunotherapy and hypophysitis: Clinical presentation, treatment, and biologic insights. Pituitary 19, 82-92. https://doi. org/10.1007/s11102-015-0671-4 (2016).

17. Joshi, M. N., Whitelaw, B. C., Palomar, M. T., Wu, Y. \& Carroll, P. V. Immune checkpoint inhibitor-related hypophysitis and endocrine dysfunction: Clinical review. Clin Endocrinol (Oxf) 85, 331-339. https://doi.org/10.1111/cen.13063 (2016).

18. Ohara, N. et al. Isolated adrenocorticotropic hormone deficiency and thyroiditis associated with nivolumab therapy in a patient with advanced lung adenocarcinoma: A case report and review of the literature. J. Med. Case Rep. 13, 88. https://doi.org/10.1186/ s13256-019-2002-2 (2019).

19. Faje, A. et al. Hypophysitis secondary to nivolumab and pembrolizumab is a clinical entity distinct from ipilimumab-associated hypophysitis. Eur. J. Endocrinol. 181, 211-219. https://doi.org/10.1530/EJE-19-0238 (2019).

20. Kobayashi, T. et al. Pituitary dysfunction induced by immune checkpoint inhibitors is associated with better overall survival in both malignant melanoma and non-small cell lung carcinoma: A prospective study. J. Immunother. Cancer 8, e000779. https://doi. org/10.1136/jitc-2020-000779 (2020).

21. Inaba, H. et al. Comparative analysis of human leucocyte antigen between idiopathic and anti-PD-1 antibody induced isolated adrenocorticotropic hormone deficiency: A pilot study. Clin. Endocrinol. (Oxf.) 91, 786-792. https://doi.org/10.1111/cen.14082 (2019).

22. Yano, S. et al. Human leucocyte antigen DR15, a possible predictive marker for immune checkpoint inhibitor-induced secondary adrenal insufficiency. Eur. J. Cancer 130, 198-203. https://doi.org/10.1016/j.ejca.2020.02.049 (2020).

23. Akamizu, T. et al. Association of autoimmune thyroid disease with microsatellite markers for the thyrotropin receptor gene and CTLA-4 in Japanese patients. Thyroid 10, 851-858. https://doi.org/10.1089/thy.2000.10.851 (2000).

24. Ryder, M., Callahan, M., Postow, M. A., Wolchok, J. \& Fagin, J. A. Endocrine-related adverse events following ipilimumab in patients with advanced melanoma: A comprehensive retrospective review from a single institution. Endocr. Relat. Cancer 21, 371-381. https://doi.org/10.1530/ERC-13-0499 (2014).

25. Chang, L. S. et al. Endocrine toxicity of cancer immunotherapy targeting immune checkpoints. Endocr. Rev. 40, 17-65. https:// doi.org/10.1210/er.2018-00006 (2019).

26. Levy, M. J., Matharu, M. S., Meeran, K., Powell, M. \& Goadsby, P. J. The clinical characteristics of headache in patients with pituitary tumours. Brain 128, 1921-1930. https://doi.org/10.1093/brain/awh525 (2005). 
27. Jackson, R. S., Carter, G. D., Wise, P. H. \& Alaghband-Zadeh, J. Comparison of paired short Synacthen and insulin tolerance tests soon after pituitary surgery. Ann. Clin. Biochem. 31(Pt 1), 46-49. https://doi.org/10.1177/000456329403100108 (1994).

28. Mukherjee, J. J. et al. A comparison of the insulin tolerance/glucagon test with the short ACTH stimulation test in the assessment of the hypothalamo-pituitary-adrenal axis in the early post-operative period after hypophysectomy. Clin. Endocrinol. (Oxf.) 47, 51-60. https://doi.org/10.1046/j.1365-2265.1997.2151035.x (1997).

29. Dokmetas, H. S. et al. A comparison between the 1-microg adrenocorticotropin (ACTH) test, the short ACTH (250 microg) test, and the insulin tolerance test in the assessment of hypothalamo-pituitary-adrenal axis immediately after pituitary surgery. J. Clin. Endocrinol. Metab. 85, 3713-3719. https://doi.org/10.1210/jcem.85.10.6879 (2000).

\section{Acknowledgements}

This work was supported by a grant-in-aid for scientific research from the Ministry of Education, Science, Sports, and Culture, Japan (19K08996). We acknowledge all members of the Immune-Checkpoint Inhibitors Working Group at The University of Tokyo Hospital. We also acknowledge the doctors and supporting medical stuff who treated and cared for the patients analyzed in this study.

\section{Author contributions}

N.M. designed the study. K.M. performed this clinical study and analyzed the data. K.M., N.M. and T.I. wrote this manuscript. All authors provided critical feedback for intellectual content of manuscript and approved the final version of the manuscript for publication.

\section{Competing interests}

The authors declare no competing interests.

\section{Additional information}

Correspondence and requests for materials should be addressed to N.M.

Reprints and permissions information is available at www.nature.com/reprints.

Publisher's note Springer Nature remains neutral with regard to jurisdictional claims in published maps and institutional affiliations.

(c) (i) Open Access This article is licensed under a Creative Commons Attribution 4.0 International License, which permits use, sharing, adaptation, distribution and reproduction in any medium or format, as long as you give appropriate credit to the original author(s) and the source, provide a link to the Creative Commons licence, and indicate if changes were made. The images or other third party material in this article are included in the article's Creative Commons licence, unless indicated otherwise in a credit line to the material. If material is not included in the article's Creative Commons licence and your intended use is not permitted by statutory regulation or exceeds the permitted use, you will need to obtain permission directly from the copyright holder. To view a copy of this licence, visit http://creativecommons.org/licenses/by/4.0/.

(C) The Author(s) 2021 